\title{
BRAQUITERAPIA DE ALTA TAXA DE DOSE NO TRATAMENTO DO CARCINOMA DA PRÓSTATA: ANÁLISE DA TOXICIDADE AGUDA E DO COMPORTAMENTO BIOQUÍMICO*
}

\author{
Sérgio Carlos Barros Esteves ${ }^{1}$, Antonio Carlos Zuliani de Oliveira ${ }^{1}$, Herbeni Cardoso ${ }^{1}$, \\ Eduardo Komai Tagawa ${ }^{1}$, Márcio D'Império ${ }^{2}$, Roberto Castelo ${ }^{3}$
}

Resumo OBJETIVO: Analisar a resposta bioquímica nas variáveis volume prostático, valor do antígeno prostático específico (PSA), escores de Gleason, estádio, risco da doença e hormonioterapia. MATERIAIS E MÉTODOS: No período de fevereiro de 1998 a julho de 2001, 46 pacientes com câncer de próstata foram tratados com radioterapia, numa combinação de teleterapia e braquiterapia de alta taxa de dose (BATD). A idade variou de 51 a 79 anos (média de 66,4 anos). 0 estádio T1c foi o mais freqüente: 30 (65\%). 0 escore de Gleason era abaixo de $7 \mathrm{em} 78 \%$ dos pacientes. O PSA variou de 3,4 a 33,3, estando abaixo de $10 \mathrm{em} 39 \%$ das vezes. $O$ volume prostático médio foi de $32,3 \mathrm{cc}$. Um total de $28 \%$ dos pacientes recebeu hormonioterapia. A dose de teleterapia variou de 45 a 50,4 Gy, associada a quatro frações de BATD de 4 Gy. RESULTADOS: $O$ seguimento variou de 6 a 43 meses. Quatro pacientes perderam seguimento e quatro morreram (um por doença). Dos 39 pacientes analisados, 76\% apresentaram PSA menor que 1,5. Nenhuma das variáveis analisadas foi estatisticamente significante $(p>0,05)$ com relação ao controle bioquímico. CONCLUSÃO: A utilização de BATD foi eficiente no tratamento do câncer de próstata e, neste estudo, as variáveis consideradas como fatores prognósticos não interferiram no controle bioquímico.

Unitermos: Câncer de próstata; Braquiterapia; Radioterapia intersticial.

Abstract High-dose rate brachytherapy in the treatment of prostate cancer: acute toxicity and biochemical behavior analysis.

OBJECTIVE: This study focuses on the biochemical response of the following variables: prostate volume, prostate-specific antigen (PSA) value, Gleason scores, staging, the risk of the disease, and hormonotherapy. MATERIALS AND METHODS: In the period between February of 1998 and July of 2001, 46 patients with prostate cancer were treated with radiotherapy, in a combination of teletherapy and high-dose rate brachytherapy (HDR). The age ranged from 51 to 79 years (averaging 66.4 years). T1c stage was the most frequent one: $30(65 \%)$. The Gleason score was below 7 in $78 \%$ of the patients. PSA ranged from 3.4 to 33.3 , being below 10 in $39 \%$ of the cases. The average prostatic volume was $32.3 \mathrm{cc}$. Twenty-eight percent of the patients received hormonotherapy. Teletherapy dose ranged from 45 to $50.4 \mathrm{~Gy}$, associated to four fractions of 4 Gy of HDR. RESULTS: The follow-up period varied from 6 to 43 months. Four patients missed the follow-up and four died (one due to the disease). Out of the 39 patients that were analyzed, $76 \%$ presented a less than 1.5 PSA. None of the analyzed variables were found to be of statistical significance $(p>0.05)$ regarding biochemical control. CONCLUSION: The use of HDR brachytherapy was found to be effective in the treatment of prostate cancer and, in this study, the variables considered as prognostic factors did not interfere in the biochemical control.

Keywords: Prostate cancer; Brachytherapy; Interstitial radiotherapy.

\section{INTRODUÇÃO}

A braquiterapia, depois da cirurgia, foi a modalidade terapêutica de maior impacto para os tumores sólidos no início do sé-

* Trabalho realizado nos Serviços de Radioterapia e Urologia da Beneficência Portuguesa de São Paulo - Hospital São Joaquim, São Paulo, SP

1. Médicos Radioterapeutas.

2. Médico Urologista.

3. Médico Radiologista.

Endereço para correspondência: Dr. Sérgio Esteves. Departamento de Radioterapia, CAISM-Unicamp. Rua Alexander Fleming 101, Cidade Universitária Zeferino Vaz. Campinas, SP, 13083 970. E-mail: estevesrt@uol.com.br.

Recebido para publicação em 30/4/2004. Aceito, após revisão, em 17/6/2005. culo 20. Naquele momento, os equipamentos de teleterapia (radioterapia externa) eram de baixa energia e não conseguiam doses adequadas na profundidade. Enquanto isso, a braquiterapia proporcionava altas doses concentradas em volumes restritos, fazendo com que as estruturas normais adjacentes recebessem baixas doses de radiação.

O primeiro implante de próstata nos Estados Unidos foi no Memorial Hospital, em outubro de 1915, realizado por Barringer, que utilizou agulhas de rádio colocadas através do períneo e guiadas pelo toque retal.
Havia muita dificuldade técnica em relação ao isótopo radioativo. O profissional se expunha à radiação, o decaimento produzia gases tóxicos e não se conseguia uma distribuição adequada do material em toda a próstata.

A partir da Segunda Guerra Mundial, ocorreu um grande avanço tecnológico com o surgimento de novos elementos radioativos e desenvolvimento de equipamentos com maiores energias e capacidade de penetração.

$\mathrm{O}$ acelerador linear parecia capaz de resolver o problema do tratamento do carcinoma da próstata. Porém, a dose máxima 
tolerável para se ter os níveis aceitáveis de complicações (aproximadamente 5\%) está entre 6.500 e 7.000 cGy. Com a braquiterapia pode-se alcançar o nível de dose duas vezes maior para as mesmas taxas de complicações.

Atualmente, as duas técnicas (teleterapia e braquiterapia) estão mais evoluídas. De um lado, a conformacional, com planejamentos em três dimensões, permite atingir doses mais elevadas ( 8.000 cGy) com baixa toxicidade ${ }^{(\mathbf{1})}$, e de outro, a braquiterapia, com fontes em miniatura guiadas por controle remoto, determina a maior precisão da técnica, sem qualquer exposição do médico e paramédicos à radiação.

O implante da próstata pode ser permanente ou temporário. No permanente, os isótopos mais utilizados no momento são o paládio-103 e o iodo-125. Neste estudo, foram usados implantes temporários com irídio-192, utilizando equipamento de braquiterapia de alta taxa de dose (BATD) associada à teleterapia (conformacional).

Vários centros mundiais, como Kiel, na Alemanha, Seatle, em Washington, Royal Oak, em Michigan e Long Beach, na Califórnia, passaram a utilizar a BATD no tratamento do carcinoma da próstata a partir dos anos $80^{(2)}$. No Brasil, alguns serviços já trataram centenas de pacientes com o método.

O objetivo deste estudo foi avaliar a taxa de resposta bioquímica do antígeno prostático específico (PSA), a influência de diversas variáveis (idade, volume da próstata, PSA, Gleason, estadiamento, risco, hormonioterapia) no controle bioquímico e as complicações agudas e subagudas dos procedimentos de acordo com a graduação da Radiation Therapy Oncology Group $(\mathrm{RTOG})^{(3)}$.

\section{MATERIAIS E MÉTODOS}

Este estudo clínico, de caráter prospectivo, incluiu pacientes portadores de carcinoma da próstata com expectativa de vida $\geq 10$ anos e Karnofsky $\geq 70^{(4)}$, estádio clínico $\mathrm{T}_{1}$ a $\mathrm{T}_{3}$, com exames de estadiamento e volume prostático $\leq 60 \mathrm{~cm}^{3}$. Foram excluídos os pacientes submetidos a tratamento prévio (radioterapia completa e/ou cirurgia), estádio clínico $\mathrm{T}_{4}$, volume prostático $>60 \mathrm{~cm}^{3}$ e Karnofsky $<70$.
No período de fevereiro de 1998 a julho de 2001, 46 pacientes foram tratados com radioterapia, associando-se teleterapia com BATD.

A idade variou de 51 a 79 anos (média de 66,4 anos). Trinta pacientes foram estadiados como T1c, sete como T2a, oito como T2b e um como T3a.

Todos os pacientes apresentaram como tipo histológico o adenocarcinoma, com a seguinte distribuição do valor do Gleason (somatório): Gleason 2, um paciente; Gleason 3, dois pacientes; Gleason 4, seis pacientes; Gleason 5, dez pacientes; Gleason 6, 17 pacientes; Gleason 7, sete pacientes; Gleason 8, dois pacientes; o Gleason ficou desconhecido em apenas um paciente. $\mathrm{O}$ PSA inicial variou de 3,4 a 33,1.

O volume da próstata pré-tratamento variou de 15 a 67 (volume médio = 32,3 cc). Treze pacientes foram submetidos a tratamento hormonal prévio (apenas como neo-adjuvância), sendo bloqueio central em três pacientes, periférico em nove e completo em três.

Considerando-se o risco, de acordo com a Tabela 1, oito foram classificados como alto risco, 16 como risco intermediário e 12 como baixo risco $^{(\mathbf{5 , 6})}$.

Tabela 1 Classificação do risco para carcinoma de próstata.

\begin{tabular}{|c|c|c|}
\hline $\begin{array}{l}\text { Baixo } \\
\text { risco }\end{array}$ & $\begin{array}{c}\text { Risco } \\
\text { intermediário }\end{array}$ & $\begin{array}{l}\text { Alto } \\
\text { risco }\end{array}$ \\
\hline $\mathrm{T} 1, \mathrm{~T} 2 \mathrm{a} \mathrm{e}$ & T2b ou & $>\mathrm{T} 2 \mathrm{~b}$ ou \\
\hline PSA $<10$ e & $\mathrm{PSA}=10-20 \mathrm{ou}$ & PSA $>20$ ou \\
\hline Gleason $<7$ & Gleason $=7$ & Gleason $>7$ \\
\hline
\end{tabular}

A dose de radiação externa foi de 5.040 cGy em 28 aplicações de 180 cGy, usandose acelerador linear de $6 \mathrm{MV}$ de energia de fótons e técnica de tratamento conformacional. Realizaram-se seis campos de tratamento diários com colimadores "multifolhas" ou colimadores próprios (Figura 1).

Para o implante de braquiterapia, o paciente é internado um dia antes e submetido a tricotomia e enteroclisma.

O paciente recebe bloqueio anestésico (raquianestesia) e é colocado em posição de semilitotomia. Realiza-se exame prostático sob anestesia e ultra-som transretal em planos sagital e coronal para avaliar a presença de lesões e determinação do vo- lume prostático. Segue-se a assepsia e colocação de sonda vesical Foley 14-16.

$\mathrm{O}$ agulhamento é guiado pelo ultra-som transretal e pelo "template" perineal. A distribuição das agulhas é realizada em todo o volume prostático de forma simétrica, identificando-se agulha por agulha, bem como sua extensão no interior da próstata.

Após a colocação das agulhas, o "template" é fixado com pontos de sutura no períneo do paciente.

Marcadores são inseridos nas agulhas e são feitas radiografias em ântero-posterior e perfil (Figura 2).

Os dados são levados para o computador, onde se calcula a melhor distribuição das fontes e a dose a ser dada no volumealvo (Figura 3).

As agulhas são conectadas ao equipamento de braquiterapia e aplicadas quatro frações de 400 cGy, com intervalo mínimo de seis horas. O paciente recebe antibiótico e analgésicos (Figura 4).

Ao término do tratamento, o "template" é retirado com as agulhas, com compressão local para hemostasia.

A braquiterapia foi realizada entre 10 e 15 dias após o término da teleterapia, com exceção de dois pacientes, que foram submetidos à braquiterapia antes da teleterapia.

O volume do implante (volume-alvo) de braquiterapia foi menor que $50 \mathrm{cc}$ em 43 pacientes $(93,5 \%)$ e maior que $50 \mathrm{cc} \mathrm{em}$ três pacientes $(6,5 \%)$.

A análise da resposta bioquímica iniciou-se dentro de um mês após o término da radioterapia e posteriormente a cada três meses no primeiro ano, passando para quatro meses no segundo ano. Para os pacientes que evoluíram com PSA satisfatório, o controle passou a ser semestral.

As variáveis analisadas em relação ao controle bioquímico foram: idade, grau histológico (Gleason), estádio clínico, valor do PSA, volume prostático, uso de hormonioterapia e risco da doença (alto, intermediário e baixo).

A análise descritiva foi realizada através de tabelas de freqüência para as variáveis categóricas e medidas de posição e dispersão para as variáveis contínuas. Para comparar proporções ou verificar associação, foi utilizado o teste exato de Fisher. 


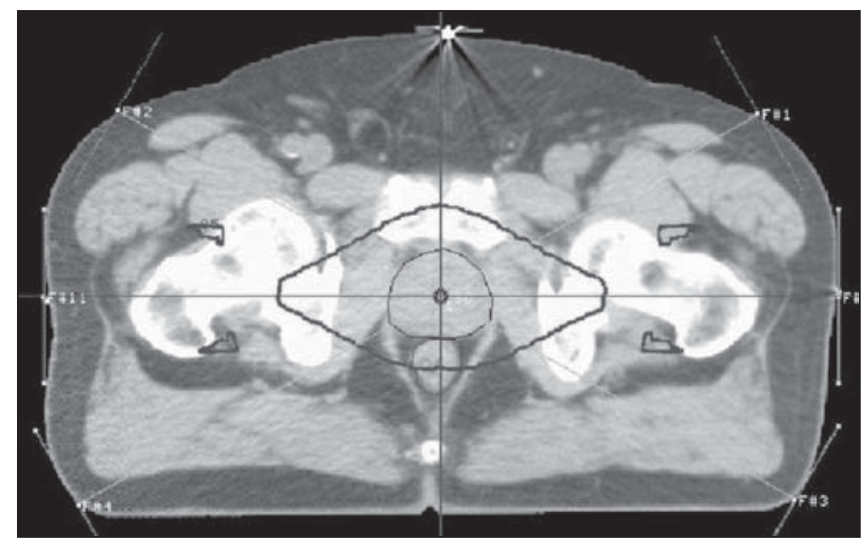

A

Figura 1. A,B: Curvas de isodose no tratamento conformacional seis campos.

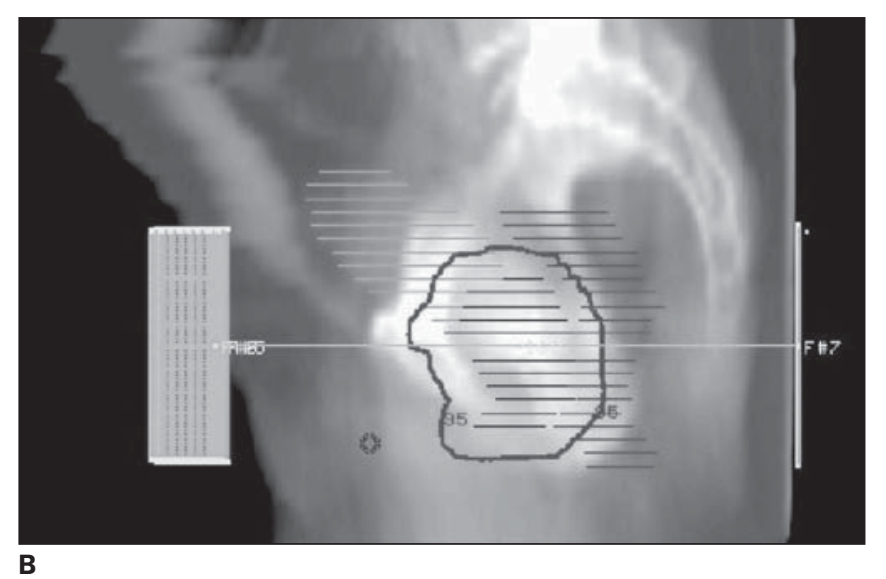

B
Figura 2. A,B: Radiografia do implante.

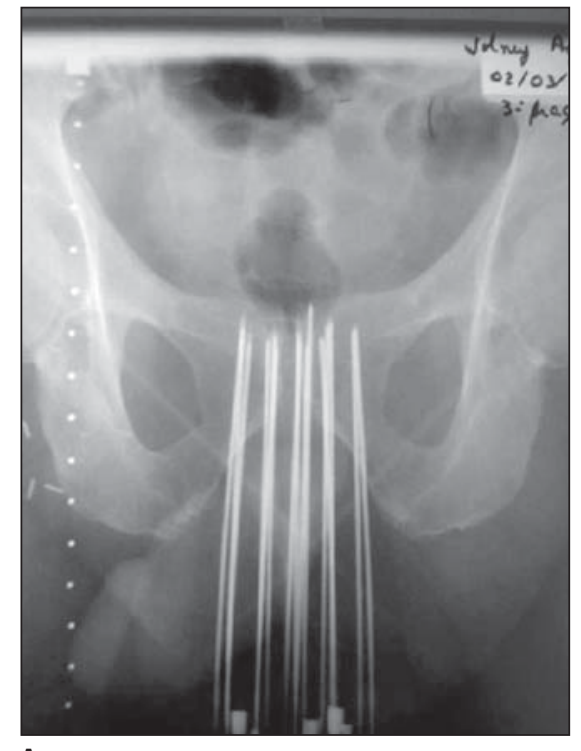

A

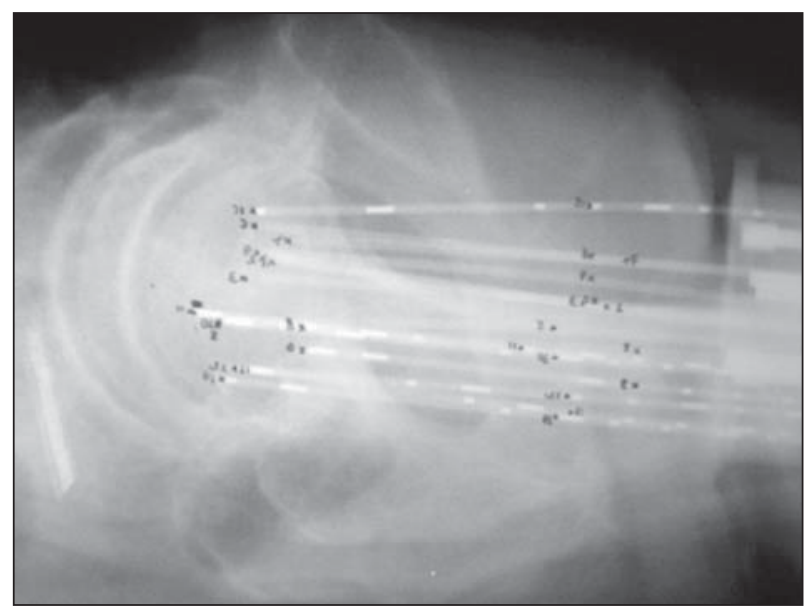

B

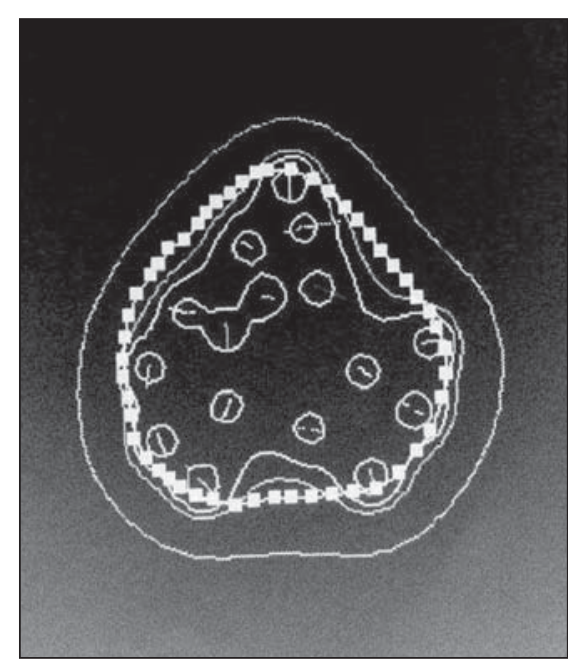

A

Figura 3. A,B: Curvas de isodose da braquiterapia.

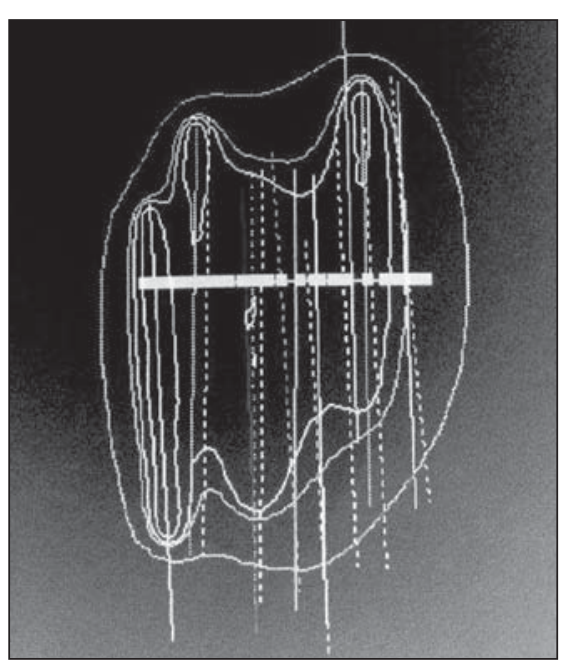

B

Para comparar medidas contínuas ou ordenáveis entre dois grupos, foi utilizado o teste de Mann-Whitney. O nível de significância adotado foi de $5 \%$.

\section{RESULTADOS}

O seguimento variou de 6 a 43 meses. Dos 46 pacientes tratados com esta técnica de tele e braquiterapia, 38 fizeram seguimento adequado com avaliação do PSA. Quatro pacientes perderam o seguimento e quatro faleceram, sendo um pela doença, um por complicações cardíacas e dois por causas desconhecidas.

Entre os 39 pacientes analisados, 26 tiveram nadir do PSA abaixo de 1,0, três ficaram com o PSA entre 1,0 e 1,5, três 


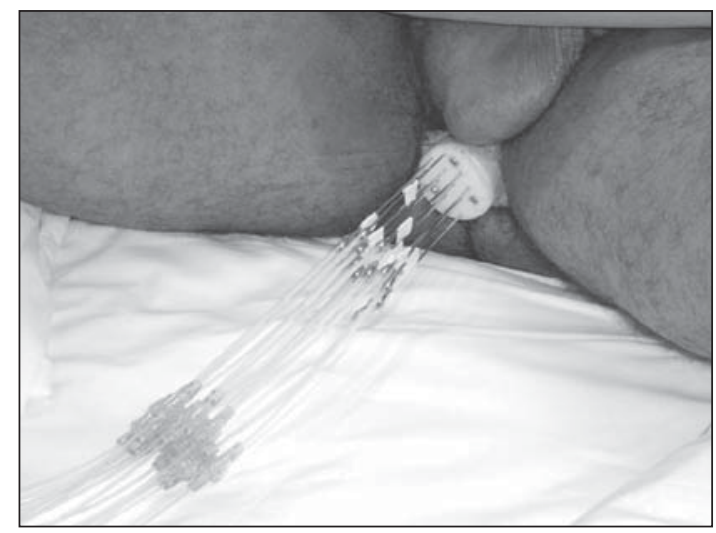

A

entre 1,5 e 2,0, seis com PSA maior que 2,0 (entre esses, um chegou a ter PSA $<1,0$ ).

Considerando o PSA $<1,5$, o controle bioquímico atual está em 76,32\% dos pacientes tratados com radioterapia.

Entre as variáveis analisadas, nenhuma delas se mostrou estatisticamente significativa em relação ao controle bioquímico, conforme mostra a Tabela 2.

Apenas três pacientes apresentaram toxicidade para o aparelho geniturinário, com grau I em dois pacientes $(5,1 \%)$ e grau II em um paciente $(2,5 \%)$.

\section{DISCUSSÃO}

A BATD é tratamento consagrado na literatura para a terapêutica dos tumores ginecológicos ${ }^{(7)}$ e sua eficácia vem sendo comprovada para outros tumores como os da próstata ${ }^{(8)}$. Estudos recentes avaliando efeitos biológicos da radiação mostram que a alta taxa de dose pode ser superior à baixa taxa de dose, dependendo do tecido alvo ${ }^{(\mathbf{9})}$.

A facilidade do método, o baixo custo quando comparado ao implante permanente e sua versatilidade devem ser considerados como vantagens sobre as outras abordagens terapêuticas.

O procedimento é muito bem tolerado pelo paciente e as complicações inerentes ao tratamento são muito pequenas.

O grupo estudado teve idade média de 66,4 anos, sendo o paciente mais idoso com 69 anos. Este dado mostra que, mesmo com idades mais avançadas, a tolerância é muito boa.

A maioria dos pacientes $(65,2 \%)$ foi estadiada como T1c, fato importante na se-

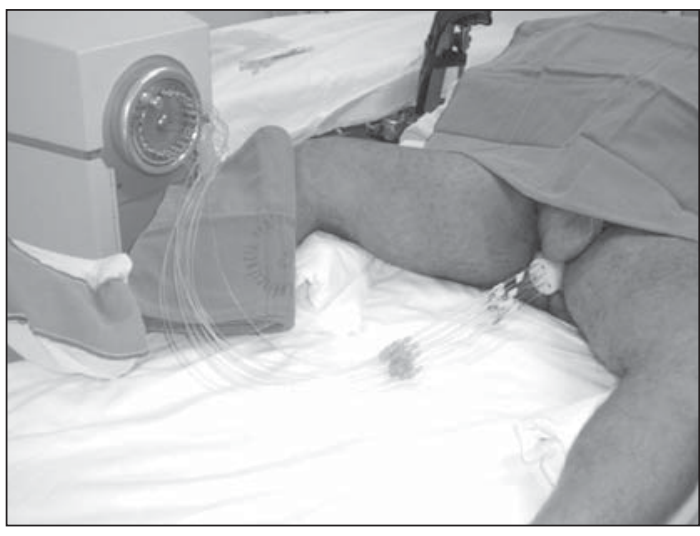

Figura 4. A,B: Paciente em posição de tratamento (BATD).
Tabela 2 Análise estatística das diferentes variáveis estudadas em relação ao controle do PSA.

\begin{tabular}{|l|c|}
\hline \multicolumn{2}{|c|}{ Controle bioquímico (PSA < 1,5). } \\
\hline Variável & $p$-valor \\
\hline Idade & 0,17 \\
Volume (braquiterapia) & 0,11 \\
PSA & 1,00 \\
Gleason & 0,80 \\
Estadiamento & 0,84 \\
Risco (alto, médio e baixo) & 0,85 \\
Hormonioterapia & 0,12 \\
\hline
\end{tabular}

leção dos pacientes, pois o subestadiameto é relativamente frequiente, haja vista os achados de anatomopatológico. Certamente, entre estes pacientes estadiados como T1c existem aqueles com doenças mais avançadas.

Oitenta por cento dos pacientes apresentaram Gleason < 7. Esta situação é boa para a BATD, pois tumores de baixo grau histológico sofrem maior efeito biológico da BATD $^{(9)}$.

Como fator isolado, o PSA esteve abaixo de 10 em $53 \%$ dos pacientes tratados. Nestes espera-se menor probabilidade de disseminação da doença. Considerando os outros fatores (estádio e Gleason), estratificamos os pacientes em baixo risco, risco intermediário e alto risco. Notamos que a maioria é de risco intermediário (44\%), enquanto $33 \%$ são de risco baixo e $22 \%$ são de alto risco. Os resultados poderiam ser mais satisfatórios se tivéssemos maior número de pacientes no baixo risco ${ }^{(\mathbf{1 0})}$.

Todas as variáveis analisadas não mostraram nenhuma significância em relação ao controle bioquímico. Este resultado pode estar relacionado ao pequeno número de pacientes estudados, como também ao intervalo de seguimento médio muito curto para a doença em estudo.

\section{REFERÊNCIAS}

1. Bagshaw MA, Cox RS, Ramback JE. Radiation therapy for localized prostate cancer. Justification by long-term folow-up. Urol Clin North Am 1990; 17:787-802.

2. Khan K, Thompson W, Bush S, Stidley C. Transperineal percutaneous iridium-192 interstitial template implant of the prostate: results and complications in 321 patients. Int J Radiat Oncol Biol Phys 1992;22:935-939.

3. Rubin $P$, Wasserman TH. International Clinical Trials in Radiation Oncology. The late effects of toxicity scoring. Int J Radiat Oncol Biol Phys 1988; 14(Suppl 1):S29-38.

4. Schaafsma J, Osoba D. The Karnofsky Performance Status Scale re-examined: a cross-validation with the EORTC-C30. Qual Life Res 1994;3: 413-424.

5. Zagars GK, Pollack A, von Eschenbach AC. Prostate cancer and radiation therapy - the message conveyed by serum prostate-specific antigen. Int $\mathbf{J}$ Radiat Oncol Biol Phys 1995;33:23-35.

6. Zagars GK, Sherman NE, Babaian RJ. Prostatespecific antigen and external beam radiation therapy in prostate cancer. Cancer 1991;67:412-420.

7. Arai T, Nakano T, Morita S, Sakashita K, Nakamura YK, Fukuhisa K. High-dose-rate remote afterloading intracavitary radiation therapy for cancer of the uterine cervix. A 20-year experience. Cancer 1992;69:175-180.

8. Martinez A, Gonzalez J, Stromberg J, et al. Conformal prostate brachytherapy: initial experience of a phase I/II dose-escalating trial. Int J Radiat Oncol Biol Phys 1995;33:1019-1027.

9. Orton CG. High-dose-rate brachytherapy may be radiobiologically superior to low-dose rate due to slow repair of late-responding normal tissue cells. Int J Radiat Oncol Biol Phys 2001;49:183-189.

10. Kuban DA, el-Mahdi AM, Schellhammer PF. Prostate-especific antigen for pretreatment prediction and posttreatment evaluation of outcome after definitive irradiation for prostate cancer. Int J Radiat Oncol Biol Phys 1995;32:307-316. 